\title{
IMPRESSÕES LINGÜÍSTICAS SOBRE DUAS AXIOMATIZAÇões PARA A Gramática CATEGoriaL ${ }^{*}$ Linguistic impressions on two axiomatizations for Categorial Grammar
}

\author{
Luiz Arthur Pagani**
}

INTRODUÇÃo

No presente texto, ${ }^{1}$ discute-se de um ponto de vista exclusivamente lingüístico duas axiomatizações ligeiramente diferentes da Gramática Categorial, mas que são logicamente equivalentes. Numa dessas versões, a gramática é definida por um conjunto de seis pares de regras de redução (aplicação, permutação, ${ }^{2}$ composição, promoção, ${ }^{3}$ divisão para o funtor prin-

* o presente texto foi preparado no sistema LATEX, por meio de sua implementação para Linux - o TeTEX - e de um programa de integração (IDE) - o Kile -, tudo isso instalado em computadores funcionando com o sistema operacional Kurumin, dentro das diretrizes do chamado software livre. Expresso assim o meu reconhecimento à enorme equipe anônima que desenvolve todos esses recursos.

** UFPR.

1 Agradeço a meus companheiros do Laboratório de Lingüística, Lógica e Computação, da Universidade Federal do Paraná, por oferecer um ambiente propício ao estudo e à discussão de questões relacionadas à Gramática Categorial; agradeço em especial a Rodrigo Tadeu Gonçalves por algumas sugestões diretas sobre o presente texto. Uma versão anterior foi apresentada no II Congresso sobre Tecnologia da Informação e da Linguagem (TIL), ocorrido em Salvador, em 2004.

2 Normalmente, o termo usado aqui é "associatividade", do inglês associativity, mas, seguindo uma observação de Oehrle, citada em Wood (1993, p. 37), prefiro usar o termo "permutação", traduzindo o inglês swapping, que remete à troca da ordem em que os argumentos se combinam com seu funtor.

3 A escolha desse termo ainda é mais complicada do que a do anterior, porque em inglês o mesmo conceito é mencionado em mais de um termo: raising (WOOD, 1993, p. 42), lifting (MOORTGAT, 1988, p. 11) e shifting (CARPENTER, 1997, p. 100). Preferi então o termo "promoção", mas em Borges Neto (1999) o termo usado é "elevação"; só acho inadequado o uso de "alçamento", porque esse termo tem sido usado na Gramática Gerativa para designar um outro tipo de fenômeno (o movimento de um constituinte para uma posição mais alta na árvore de estrutura sintagmática da expressão). 
cipal e divisão para o funtor subordinado) que associa certas operações semânticas às respectivas operações de combinação categorial; na segunda versão, essas mesmas regras são teoremas deriváveis a partir de dois pares de regras de inferência (ou dedução): uma regra de introdução e outra de eliminação para cada conectivo categorial (/ e $\mid){ }^{4}$

Nesta apresentação, no entanto, não nos deteremos no aspecto lógico ou dedutivo das axiomatizações. O principal objetivo aqui não é discutir a decidibilidade ou a precisão axiomática de cada um dos dois sistemas, mas sim chamar a atenção para certos aspectos ontológicos e epistemológicos da representação do conhecimento lingüístico. Para isso, em vez de postularmos alguma espécie de primazia algébrica ou computacional de um sistema em relação ao outro (que talvez nem exista, se eles forem mesmo logicamente equivalentes), vamos decidir essa primazia a partir de determinadas características que cada um desses sistemas apresenta em relação ao tipo de explicação que ele pode sugerir para o trabalho de um lingüista.

\section{Gramáticas Categoriais}

Segundo Moortgat (1988, p. 1-2), uma Gramática Categorial se distingue de outras teorias lingüísticas muito semelhantes (tais como a Gramática de Estrutura Sintagmática Generalizada (Generalized Phrase Structure Grammar, GPSG) ou a Gramática de Estrutura Sintagmática Conduzida pelo Núcleo (Head-Driven Phrase Structure Grammar, HPSG)) por apresentar as seguintes quatro características:

\section{Lexicalismo}

As teorias gramaticais que se concentram na estrutura superficial compartilham uma tendência em deslocar para o léxico a carga explicativa que, em outras teorias, seria atribuída ao componente sintático. Ao desenvolver uma noção mais ampla de estrutura categorial, por exemplo, a GPSG torna desnecessário um componente transformacional como o da Gramática

4 Na verdade, no cálculo de Lambek há um terceiro conectivo $(\bullet)$, o que exige mais um par de regras de inferência; no entanto, a ausência desse terceiro conectivo e de suas respectivas regras de dedução não afeta as questões discutidas no presente texto. Além disso, ambas as axiomatizações são compostas efetivamente por esquemas de regras de inferência, que ainda precisam ser preenchidos por categorias para se tornarem regras efetivamente; no entanto, tomaremos aqui a liberdade terminológica de chamá-las apenas de regras de inferência. 
Gerativa Clássica. A Gramática Categorial avança um passo a mais em direção ao lexicalismo, tornando desnecessário o próprio componente sintagmático. A informação sintática é completamente projetada a partir das estruturas categoriais atribuídas aos itens lexicais. Na sua forma mais pura, a Gramática Categorial identifica o léxico como o único local para as estipulações específicas às línguas. A sintaxe é uma álgebra livre: uma combinatória universal conduzida pelas estruturas categoriais complexas.

Estrutura de função e argumento

A contribuição categorial mais específica para a teoria das categorias é a de que as expressões incompletas são modeladas, sintática e semanticamente, como funtores. As dependências elementares entre as expressões, que determinam fenômenos como a regência, o controle e a concordância, são todas definidas por meio da hierarquia entre funções e argumentos, e não por sua configuração estrutural.

Flexibilidade dos constituintes

A Gramática Categorial Clássica, ${ }^{5}$ assim como a Gramática de Estrutura Sintagmática, atribui uma única estrutura de constituintes a uma expressão não-ambígua. As teorias categoriais generalizadas substituem essa noção de constituintes por outra mais flexível, oferecendo um inventário mais amplo de operações combinatórias que configuram um cálculo da mudança de tipo. Uma expressão não-ambígua é associada a um conjunto de derivações equivalentes. A coordenação booleana generalizada funciona como uma técnica experimental que revela os constituintes alternativos ocultos.

Composicionalidade

A relação entre a álgebra sintática e a álgebra semântica é um homomorfismo, ou seja, uma relação que preserva a estrutura, na qual cada operação sintática corresponde a uma operação semântica. A Gramática Categorial clássica incorpora uma forma de composicionalismo bastante forte, baseada na correspondência entre a regra de redução sintática central e a aplicação funcional na semântica. Os sistemas categoriais generalizados ampliam esta forma forte de composicionalidade para o cálculo da

\footnotetext{
5 O emprego que Moortgat dá a esse termo é diferente de meu emprego no texto. Moortgat se refere aqui ao que Carpenter (1997, p. 140) chama de fragmento aplicativo; meu uso é igual ao de Wood (1993, p. 34).
} 
mudança de tipo, executando assim o programa da interpretação conduzida pelo tipo. Uma conseqüência dessas características é que muitos dos fenômenos lingüísticos que exigiram da Gramática Gerativa, por exemplo, a postulação de categoria vazia, deslocamento e eliminação de estrutura, poderão ser monotonicamente resolvidos na Gramática Categorial, sem recorrer a operações destrutivas como estas.

\title{
Gramática Categorial Clássica
}

Numa de suas versões mais difundidas entre os poucos lingüistas que adotam a Gramática Categorial, normalmente ela é definida como um conjunto de seis pares de regras de redução ${ }^{6}$ que associam uma operação de combinação categorial e uma operação de construção da representação semântica.

Nessa versão, seguindo Moortgat (1988, p. 11), ${ }^{7}$ os seis pares de regras de redução são definidos da seguinte maneira:

$$
\begin{aligned}
& \text { R1 Aplicação } \\
& \text { - } X / Y: f, Y: a \Rightarrow X: f(a) \\
& \text { - } Y: a, Y \mid X: f \Rightarrow X: f(a) \\
& \text { R2 Composição } \\
& \text { - } X / Y: f, Y / Z: g \Rightarrow X / Z: \lambda v[f(g(v))] \\
& \text { - } Z|Y: g, Y| X: f \Rightarrow Z \mid X: \lambda v[f(g(v))] \\
& \text { R3 Permutação } \\
& \text { - }(Z \mid X) / Y: f \Rightarrow Z \mid(X / Y): \lambda v_{1}\left[\lambda v_{2}\left[f\left(v_{2}\right)\left(v_{1}\right)\right]\right] \\
& \text { - } Z \mid(X / Y): f \Rightarrow(Z \mid X) / Y: \lambda v_{1}\left[\lambda v_{2}\left[f\left(v_{2}\right)\left(v_{1}\right)\right]\right]
\end{aligned}
$$

\section{R3 Permutação}

\begin{abstract}
- Na verdade, esta é a versão mais ampla dessa vertente. A primeira versão da Gramática Categorial, proposta por Ajdukiewicz (1935), tinha apenas uma das regras de aplicação, porque ela não era direcional; numa das primeiras aplicações mais lingüisticamente motivadas (BAR-HILLEL, 1953), conhecida como modelo AB, apenas o par de regras de aplicação era usado; numa outra versão, conhecida como Gramática Categorial Livre (COHEN, 1967), além das regras de aplicação, aparecem também as regras de composição, de permutação e de promoção. No entanto, o modelo mais empregado modernamente é mesmo esse de seis pares de regras, que também aparece num formato um pouco alterado na formulação de Steedman (1988), chamada de Gramática Categorial Combinatória.

As expressões do cálculo- $\lambda$ que representam a interpretação semântica receberam uma notação um pouco mais explícita aqui. No texto original, o escopo do operador- $\lambda$ era marcado com um ponto, como em $\lambda$ P.P (x), o que é bastante usual; no entanto, como em fórmulas muito longas pode ficar difícil perceber o escopo do operador, preferi uma notação na qual o escopo recebe um marcador de início e de fim, como em $\lambda \mathrm{P}[\mathrm{P}(\mathrm{x})]$ (onde os colchetes marcam inequivocamente o começo e o final do escopo do operador).
\end{abstract}




\section{R4 Promoção}

- $X: a \Rightarrow Y /(X \mid Y): \lambda v[v(a)]$

- $X: a \Rightarrow(Y / X) \mid Y: \lambda v[v(a)]$

R5 Divisão para o funtor principal

- $X / Y: f \Rightarrow(X / Z) /(Y / Z): \lambda v_{1}\left[\lambda v_{2}\left[f\left(v_{1}\left(v_{2}\right)\right)\right]\right]$

- $Y|X: f \Rightarrow(Z \mid Y)|(Z \mid X): \lambda v_{1}\left[\lambda v_{2}\left[f\left(v_{1}\left(v_{2}\right)\right)\right]\right]$

R6 Divisão para o funtor subordinado

- $X / Y: f \Rightarrow(Z / X) \mid(Z / Y): \lambda v_{1}\left[\lambda v_{2}\left[v_{1}\left(f\left(v_{2}\right)\right)\right]\right]$

- $Y \mid X: f \Rightarrow(Y \mid Z) /(X \mid Z): \lambda v_{1}\left[\lambda v_{2}\left[v_{1}\left(f\left(v_{2}\right)\right)\right]\right]$

De acordo com estas regras, e considerando que as expressões "Pedro", "ama" e "Maria" correspondem respectivamente aos pares de categoria sintática e representação semântica ' $\mathrm{N}: \mathrm{p}$ ', '( $\mathrm{N} \backslash \mathrm{S}) / \mathrm{N}:$ : e ' $\mathrm{N}: \mathrm{m}$ ', podemos representar a estrutura da sentença "Pedro ama Maria” em um diagrama como o da Figura $1 .^{8}$

O diagrama da Figura 1 representa a demonstração de que a sentença "Pedro ama Maria" é uma sentença que denota a relação de amar que se estabelece de Pedro para Maria ('S : A(m)(p)'), a partir das regras de redução e de três premissas:

1. "Pedro" é um nome que denota o indivíduo Pedro ('N : p’),

\begin{tabular}{|c|c|c|}
\hline \multirow{3}{*}{$\begin{array}{l}\text { Pedro } \\
\overline{N: p}^{L x}\end{array}$} & ama & Maria \\
\hline & $\overline{(N \backslash S) / N: A}^{L x}$ & $\overline{N: m}^{L x}$ \\
\hline & \multicolumn{2}{|c|}{$N \backslash S: A(m)$} \\
\hline \multicolumn{3}{|c|}{$S: A(m)(p)$} \\
\hline
\end{tabular}

Figura 1: Derivação de "Pedro ama Maria" apenas com R1.

8 Nestes diagramas, conhecidos como dedução ao estilo de Prawitz, as barras horizontais relacionam uma conclusão e suas premissas, de forma que a conclusão aparece debaixo da barra e as premissas sobre ela; ao lado direito da barra registra-se a regra empregada na inferência. No entanto, a inserção dos itens lexicais não segue bem esse padrão: as expressões lingüísticas aparecem acima das suas respectivas barras na primeira linha do diagrama, e debaixo delas são registradas suas respectivas categorias e representações semânticas. No cálculo de Lambek, que também utiliza esse tipo de diagrama, algumas derivações vão exigir ainda a introdução de suposições, que são apresentadas entre colchetes numerados com um índice, que marca o escopo entre a sua introdução e a sua eliminação. 
2. “ama” é um predicado de dois lugares que denota a relação de $\operatorname{amar}\left({ }^{(}(\mathrm{N} \backslash \mathrm{S}) / \mathrm{N}: \mathrm{A}^{\prime}\right)$,

3. “Maria” é um nome que denota o indivíduo Maria ('N : m’).

Nesse sentido, esse diagrama não é muito diferente de um diagrama em árvore, como os que são associados a uma Gramática de Estrutura Sintagmática. A grande diferença, no entanto, é que, a partir de uma Gramática de Estrutura Sintagmática para "Pedro ama Maria", conseguiríamos construir uma única árvore para essa sentença; já com uma Gramática Categorial, como a apresentada acima, poderíamos chegar a uma outra estrutura para a mesma sentença, chegando à mesma representação semântica final, por uma seqüência de regras diferente da anterior, como podemos ver no diagrama da Figura $2 .{ }^{9}$

Poderíamos chegar ainda a um terceiro diagrama para a mesma sentença "Pedro ama Maria", como na Figura 3, também com a mesma representação semântica. Na verdade, na Gramática Categorial, podemos encontrar um número infinito de derivações equivalentes para a mesma expressão, o que normalmente é chamado de ambigüidade espúria, que já foi considerada uma das principais falhas da Gramática Categorial, mas que pode ser facilmente controlada por uma exigência de normalização das derivações (como proposto por CARPENTER , 1997, p. 160-164).

No entanto, do ponto de vista lingüístico, pode-se perceber uma pequena diferença entre o diagrama da Figura 1, por um lado, e os diagramas das Figuras 2 e 3, por outro: se considerarmos que as operações realizadas pelas regras de redução equivalem também a concatenações das seqüências fonológicas, na Figura 1 teríamos uma estrutura prosódica correspondente a "(Pedro (ama Maria))" (onde os parênteses encerram os constituintes prosódicos concatenados), enquanto nas Figuras 2 e 3 teríamos a seguinte estrutura prosódica: '((Pedro ama) Maria)'.

9 A redução- $\beta$ é uma das principais operações do cálculo- $\lambda$, e pode ser caracterizada pela seguinte fórmula: $\lambda \mathrm{v}[\mathrm{F}](\mathrm{a}) \Rightarrow \mathrm{F}[\mathrm{v} \rightarrow \mathrm{a}]$, que pode ser lida como "um termo- $\lambda(\lambda \mathrm{v}[\mathrm{F}])$, aplicado a outro termo (a) é equivalente ao termo no escopo do operador com as ocorrências livres da variável v substituídas pelo termo a (F[v $\rightarrow$ a])" (CARPENTER, 1997, p. 50). 
PAGANI, L. A. Impressões Lingüísticas Sobre duAs aXiomatizaçốEs...

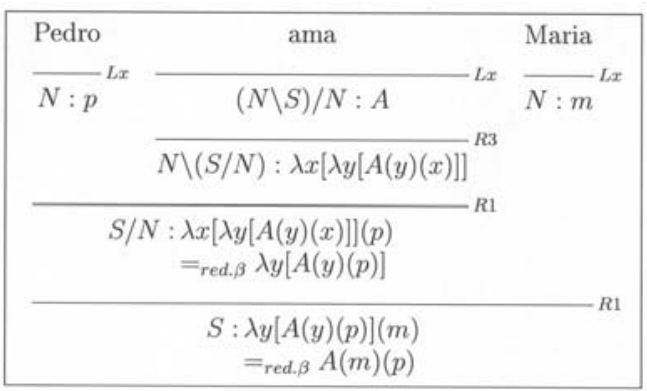

Figura 2: Derivação de "Pedro ama Maria" com R1 e R3.

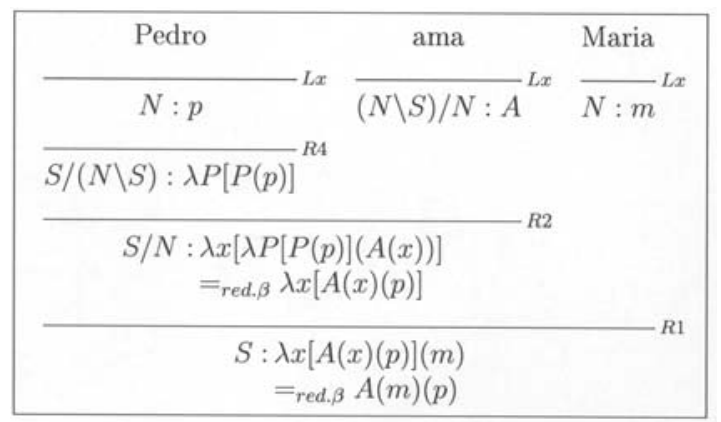

Figura 3: Derivação de "Pedro ama Maria" com R1, R2 e R4.

Apesar de semanticamente equivalentes, essas duas estruturas prosódicas apresentam características sintáticas e discursivas distintas. Por exemplo, apenas a segunda estrutura aceitaria uma continuação como "E não Márcia”, no sentido de que não é a Márcia, e sim a Maria, a pessoa que o Pedro ama; a primeira estrutura prosódica, ao contrário, é compatível com uma continuação como "E não Paulo", no sentido de que a pessoa que ama a Maria não é o Paulo, e sim o Pedro. Isso justifica, do ponto de vista lingüístico, a distinção das infinitas derivações aparentemente equivalentes de "Pedro ama Maria" em dois grupos: 1) o das que concatenam primeiro "Pedro" e "ama", e 2) o das que concatenam primeiro "ama" e "Maria".

Cálculo de Lambek em Dedução Natural

Ainda segundo Moortgat (1988, p. 2),

o cálculo de Lambek substitui o conjunto de regras de redução categorial que foram propostas na literatura (Aplicação, Composição, Promoção, etc.) por uma noção geral de derivabilidade, a par- 
tir da qual as leis de redução são consideradas teoremas. A derivabilidade é definida na forma de axiomatização de seqüentes, o que reduz as derivações categoriais a deduções lógicas com base nos procedimentos de prova desenvolvidos originalmente por Gentzen, em seu trabalho sobre o cálculo proposicional intuicionístico.

Por causa da facilidade notacional, em vez dos diagramas de derivação de seqüentes, vamos adotar aqui a mesma notação de derivação da Dedução Natural usada nos diagramas anteriores para a sentença "Pedro ama Maria”. Assim, em vez de seis pares de regras, no cálculo de Lambek, precisamos apenas de um par de regras para cada conectivo: uma de introdução e outra de eliminação do conectivo, como nos esquemas da Figura 4, abaixo, adaptados de Carpenter (1997, p. 153; 156).

$$
\frac{X / Y: f \quad Y: a}{X: f(a)} / E
$$

(a) Eliminação de /

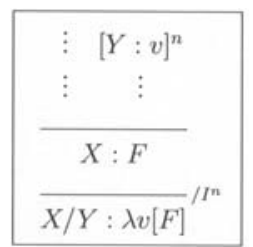

(c) Introdução de /

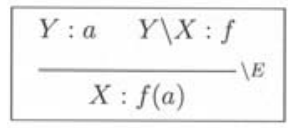

(b) Eliminação de ।

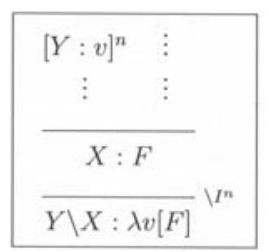

(d) Introdução de ।

Figura 4: Esquemas para eliminação e introdução dos conectivos.

Assim, nesta outra axiomatização, "as leis de redução que foram introduzidas antes como primitivos passam a ser teoremas; ou seja, inferências válidas da lógica dos conectivos categoriais" (MOORTGAT, 1988, p. 27). Como se pode perceber facilmente, o par em R1 equivale diretamente às regras de eliminação. A demonstração de R4, por exemplo, pode ser feita como na Figura 5, de forma que a partir apenas de ' $\mathrm{X}$ : a' (a outra categoria que aparece ao lado desta é apenas uma suposição) se deduz ' $\mathrm{Y} /(\mathrm{X} \backslash \mathrm{Y})$ : $\lambda \mathrm{v}[\mathrm{v}(\mathrm{a})]$ ' e ' $(\mathrm{Y} / \mathrm{X}) \backslash \mathrm{Y}: \lambda \mathrm{v}[\mathrm{v}(\mathrm{a})]$ '. Os outros quatro pares de regras podem ser igualmente demonstrados (como essas demonstrações não são essenciais aqui, elas não serão apresentadas; além do mais, elas já foram feitas por MOORTGAT, 1988). 
Em relação ao diagrama da Figura 1, com a derivação da sentença "Pedro ama Maria" apenas com a regra R1, a derivação empregando apenas os esquemas de eliminação dos conectivos, na Figura 6, não apresenta nenhuma diferença.

No entanto, o diagrama equivalente à derivação com R1 e R3 apresenta uma diferença essencial, que é a suposição de uma variável da categoria 'N', que aparece entre colchetes na derivação da Figura 7.

\begin{tabular}{|l|l|}
$\frac{X: a \quad[X \backslash Y: v]^{0}}{Y: v(a)} \backslash E$ & $\frac{[Y / X: v]^{0} \quad X: a}{Y: v(a)} / E$ \\
$\frac{Y /(X \backslash Y): \lambda v[v(a)]}{(Y / X) \backslash Y: \lambda v[v(a)]} \backslash I^{0}$ \\
\hline
\end{tabular}

Figura 5: Demonstração de R4.

\begin{tabular}{|ll|}
\hline$\frac{\text { Pedro }}{N: p} L x$ & $\frac{\text { ama }}{(N \backslash S) / N: A} L x \frac{\text { Maria }}{N: m} L x$ \\
& $\frac{S \backslash S: A(m)}{S: A(m)(p)}$ \\
\end{tabular}

Figura 6: Derivação de "Pedro ama Maria" apenas com eliminação.

\begin{tabular}{|c|c|c|}
\hline$\frac{\text { Pedro }}{N: p}^{L x}$ & $\frac{\text { ama }}{(N \backslash S) / N: A}^{L x} \quad[N: x]^{1}$ & $\frac{\text { Maria }}{N: m}_{L x}$ \\
\hline \multicolumn{3}{|c|}{$N \backslash S: A(x)$} \\
\hline \multicolumn{3}{|c|}{$S: A(x)(p)$} \\
\hline \multicolumn{3}{|c|}{$S / N: \lambda x[A(x)(p)]$} \\
\hline & $\begin{aligned} S: & \lambda x[A(x)(p)](m) \\
& ={ }_{r e d . \beta} A(m)(p)\end{aligned}$ & \\
\hline
\end{tabular}

Figura 7: Derivação de "Pedro ama Maria” equivalente à com R1 e R3.

\begin{tabular}{|c|c|c|c|c|}
\hline$\frac{\text { Pedro }}{N: p}^{L x}$ & {$[N \backslash S: P]^{1}$} & $\frac{\text { ama }}{(N \backslash S) / N: A}^{L x}$ & {$[N: x]^{2}$} & $\frac{\text { Maria }}{N: m}^{L}$ \\
\hline \multicolumn{2}{|c|}{$S: P(p)$} & \multirow{2}{*}{\multicolumn{2}{|c|}{$N \backslash S: A(x)$}} & \\
\hline \multicolumn{2}{|c|}{$S /(N \backslash S): \lambda P[P(p)]$} & & & \\
\hline \multicolumn{4}{|c|}{$\begin{aligned} S & : \lambda P[P(p)](A(x)) \\
& ={ }_{\text {red. } \beta} A(x)(p)\end{aligned}$} & \\
\hline \multicolumn{4}{|c|}{$S / N: \lambda x[A(x)(p)]$} & \\
\hline$\cdot$ & & $\begin{array}{l}\lambda x[A(x)(p)](m) \\
={ }_{r e d . \beta} A(m)(p)\end{array}$ & & \\
\hline
\end{tabular}

Figura 8: Derivação de "Pedro ama Maria" equivalente à com R1, R2 e R4. 
Para se chegar a um diagrama equivalente ao da derivação com R1, R2 e R4, na Figura 3, é necessária não apenas a suposição de uma variável que consome um dos argumentos de "ama", mas é preciso supor também uma variável que se aplique a "Pedro" para que, depois de "descarregada" a suposição, tenhamos transformado "Pedro" numa função que toma um 'N\S' para resultar num 'S', como podemos ver na Figura 8.

\section{COMPARAÇÕES}

Para um lingüista acostumado com as árvores de uma Gramática de Estrutura Sintagmática, não é difícil ver, num diagrama como o da Figura 1 , os itens lexicais claramente representados na parte superior da derivação. Logo abaixo desses itens lexicais, também são claras as representações das operações sintáticas e semânticas reunindo esses itens lexicais em expressões cada vez mais complexas. Como já foi dito, mesmo em diagramas como os das Figuras 2 e 3, com derivações incomuns para uma Gramática de Estrutura Sintagmática, podemos relacionar as regras de permutação (R3) e de promoção (R4) a uma operação que afeta a estrutura prosódica da expressão, trocando de lugar fronteiras fonológicas e reagrupando seus constituintes entoacionais.

Já em relação aos diagramas das Figuras 7 e 8 , a que tipo de coisas poderíamos relacionar as suposições entre colchetes?

Numa primeira tentativa de responder a esta pergunta, poderíamos querer ver nessas suposições as chamadas categorias vazias da Gramática Gerativa, já que elas parecem introduzir apenas uma entidade da representação semântica (uma variável que será posteriormente abstraída pelo operador- $\lambda$ ) que não corresponde a nenhuma expressão lingüística. No entanto, na Gramática Gerativa, as categorias vazias são itens lexicais. Nas derivações da Gramática Categorial, os itens lexicais são claramente representados, no topo do diagrama, como uma expressão lingüística sobre uma barra horizontal, debaixo da qual constam sua categoria sintática e sua representação semântica. É apenas na introdução dos itens lexicais que aparecem expressões lingüísticas sobre estas barras; no restante da derivação sobre as barras ficam apenas as premissas das conclusões (estas últimas localizadas sob as barras). Assim, era de se esperar que, se as suposições fossem categorias vazias, elas fossem introduzidas nos diagramas por meio de uma barra com o rótulo lexical com a parte 
acima da barra vazia; portanto, fica impossível relacionar essas suposições às categorias vazias.

Além disso, ainda que a observação a seguir possa não ter sido feita em nenhum lugar, os itens lexicais parecem corresponder a axiomas. $\mathrm{Ou}$ seja, eles precisam ser definidos em algum lugar, e eles não podem ser derivados a partir de nenhum outro teorema da Gramática Categorial. Mas, nas regras de introdução dos conectivos, as suposições não correspondem a nenhum axioma ou teorema; pelo contrário, nos diagramas a suposição nunca aparece debaixo de uma barra horizontal, como observamos. Dessa maneira, não é apenas às categorias vazias que as suposições não podem corresponder: elas também não podem ser comparadas a nenhum item lexical.

Contudo, mesmo que tivéssemos descoberto uma natureza mais lingüisticamente motivada para o papel das suposições nas regras de introdução de conectivos, ainda precisaríamos encontrar a motivação lingüística que justificasse a maior quantidade de regras empregadas nos diagramas do cálculo de Lambek, em relação aos diagramas equivalentes da versão das regras de redução.

Se observarmos os diagramas das Figuras 2 e 3, podemos constatar que ambos são formados apenas com três aplicações de regras: uma aplicação de R3 e duas de R1, no diagrama da Figura 2, e uma aplicação de R4, uma de R2 e uma de R1, no diagrama da Figura 3. E em ambos os casos é fácil relacionar as aplicações das regras unárias (R3 e R4) a operações fonológicas que afetam a organização dos constituintes prosódicos: em ambas as derivações a aplicação das regras unárias faz com que a estrutura

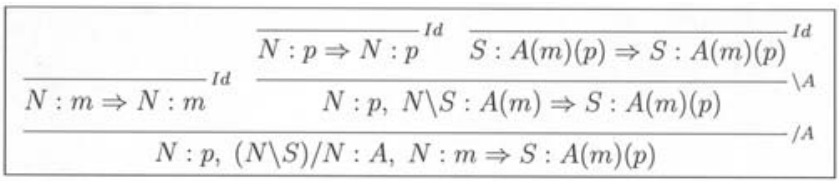

Figura 9: Derivação de "Pedro ama Maria" no Cálculo de Seqüentes prosódica se torne '((Pedro ama) Maria)'; ao contrário da derivação na qual essas regras não atuam, na Figura 1, cuja estrutura prosódica é '(Pedro (ama Maria))'.

Já nas derivações equivalentes do cálculo de Lambek, nas Figuras 7 e 8, as mesmas derivações são realizadas respectivamente com quatro e seis aplicações das regras. Aqui, fica impossível equiparar cada uma dessas aplicações das regras a qualquer operação fonológica, já que há uma mesma diferença prosódica a ser relacionada à aplicação de uma eliminação e de uma introdução, no diagrama da Figura 7, e à aplicação de duas eliminações e duas introduções, no diagrama da Figura 8. 
A diferença fica ainda mais ressaltada quando a derivação é apresentada com o cálculo de seqüentes, de Gentzen, como se pode ver na Figura $9 .{ }^{10}$

Na derivação da Figura 9, fica difícil para um lingüista reconhecer o que poderia corresponder a um item lexical, e principalmente identificar a entrada lexical relativa ao verbo "ama": como a introdução de "Maria" e de "Pedro" é feita por duas instâncias do axioma da identidade (Id), não seria de esperar que "ama" também fosse introduzido por outra instância do mesmo axioma? No cálculo de seqüentes, não. Apenas as categorias básicas aparecem nas instâncias do axioma de identidade: os nomes "Maria" e "Pedro", e a sentença "Pedro ama Maria". Como a categoria de "ama" é funcional, ele não aparece independentemente na derivação. Observe ainda que, nessa representação, as expressões lingüísticas propriamente ditas não aparecem propriamente nos diagramas: vemos apenas suas categorias e suas representações semânticas, mas não percebemos em nenhum lugar as expressões "Pedro" e "Maria".

E se já é difícil identificar os próprios itens lexicais, é ainda mais difícil relacionar com o diagrama de seqüentes as operações de concatenação prosódica apontadas anteriormente. A maneira mais simples de apresentar as derivações equivalentes às com a permutação (R3) e com promoção e composição (R4 e R2), seria demonstrando-as separadamente, e depois substituindo na derivação os itens lexicais pelas equivalências demonstradas, usando a regra de corte (cut rule). Mas a que tipo de operação lingüística poderiam corresponder essa demonstração paralela e a própria regra de corte? Com efeito, não é possível relacioná-las lingüisticamente a nada. ${ }^{11}$

\section{CoNCLUSÃo}

A partir da observação de como os itens lexicais e uma operação lingüística (a concatenação de constituintes prosódicos) poderiam ser identificados nos diagramas de derivação da Gramática Categorial, o que se conclui é que não apenas "as derivações no cálculo associativo de Lambek são representadas mais economicamente por derivações da dedução natural ao estilo de Prawitz" (MORRILL, 1994, p. 80), mas que sua versão com

\footnotetext{
10 Infelizmente, por falta de espaço, não será possível apresentar aqui a formalização do cálculo de Lambek com seqüentes; mas o leitor interessado pode consultar os livros de Moortgat (1988), Morrill (1994) e Carpenter (1997).

11 Novamente por limitação de espaço, também não apresento os diagramas de seqüentes equivalentes às derivações das Figuras 2 e 3 . Um diagrama com exemplo do uso da regra de corte para introdução da promoção do sujeito pode ser encontrado no livro de Carpenter (1997, p. 147).
} 
os seis pares de regras de redução oferece um ambiente mais propício para a reflexão mais lingüisticamente motivada.

Assim, mesmo que "a perspectiva dos seqüentes seja uma base particularmente lúcida para a discussão de questões essenciais como as de derivabilidade e de decidibilidade" (MOORTGAT, 1988, p. 27), a equivalência entre os dois sistemas garante que as descobertas feitas para a versão com os seqüentes possa ser imediatamente transferida para a Gramática Categorial Clássica, que é mais adequada ao trabalho do lingüista.

Nesse sentido, para encerrar, vamos apresentar uma adaptação para o estilo de Prawitz da representação que Morrill (1994, p. 110-129) desenvolve usando o estilo de Ficht. As principais diferenças dessa representação para as apresentadas antes são duas: 1) os itens lexicais ocupam o lugar das premissas nas demonstrações, e 2) as expressões lingüísticas aparecem explicitamente concatenadas. Os diagramas correspondentes às derivações das Figuras 1, 2 e 3 podem ser vistos, respectivamente, nas Figuras 10, 11 e 12 .

\begin{tabular}{|c|c|c|}
\hline$\overline{\text { Pedro }-N: p}^{L x}$ & $\overline{\operatorname{ama}-(N \backslash S) / N: A}^{L x}$ & $\overline{\text { Maria }-N: m}^{L x}$ \\
\hline & \multicolumn{2}{|c|}{ (ama Maria) $-N \backslash S: A(m)$} \\
\hline (Pedr & $($ ama Maria $))-S: A(n$ & $(p)$ \\
\hline
\end{tabular}

Figura 10: Explicitando a concatenação prosódica apenas com R1.

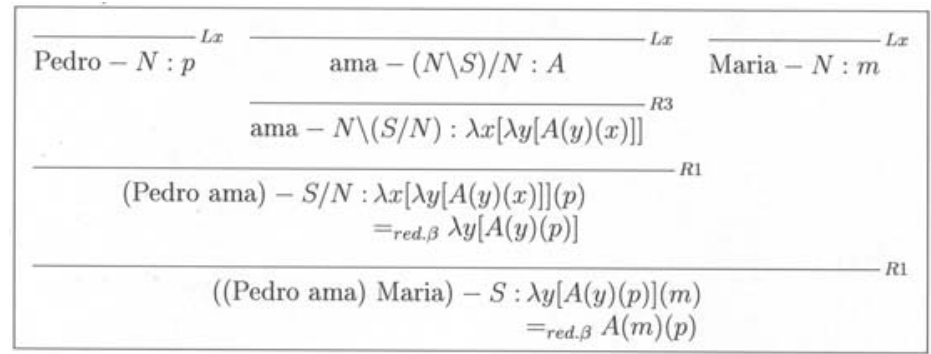

Figura 11: Explicitando a concatenação prosódica com R1 e R3.

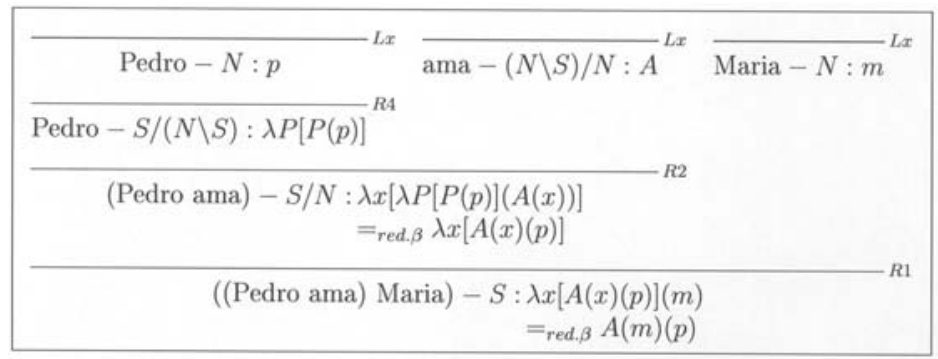

Figura 12: Explicitando a concatenação prosódica com R1, R2 e R4. 
Nestes três últimos diagramas, podemos ver claramente os dois principais pontos ressaltados durante as discussões apresentadas aqui:

1. Os itens lexicais correspondem a axiomas, introduzidos sob uma barra sem nada sobre ela; do ponto de vista lingüístico, a conseqüência é que os itens lexicais são independentes: eles não dependem diretamente de nenhuma operação da Gramática Categorial - pelo contrário, são os itens lexicais que afetam a análise lingüística representada nas derivações, assim como o lexicalismo preconiza.

2. As operações de concatenação das expressões lingüísticas estão explicitamente expressas, assim como as operações de combinação categorial e de unificação das representações semânticas; assim, em cada passo da derivação, sabemos de cada expressão lingüística construída sua categoria sintática e sua interpretação semântica, relacionando claramente expressões lingüísticas e suas respectivas interpretações semânticas, exatamente como exige a composicionalidade.

Dessa maneira, ainda que aparentemente a discussão acima tenha sido fundamentada pela ontologia das entidades lingüísticas (mais especificamente, pela natureza axiomática dos itens lexicais) e pela epistemologia das operações lingüísticas (não apenas as de combinação categorial e de interpretação semântica, mas também as de aglutinação de constituintes prosódicos), as conclusões a que acabamos de chegar afetam diretamente a representação desse tipo de conhecimento na elaboração de analisadores gramaticais que sirvam como modelo para o comportamento lingüístico humano: a implementação de analisadores para Gramáticas Categoriais que se pretendam psicologicamente realísticos deve representar os itens lexicais como axiomas de uma álgebra livre.

\section{RESUMO}

No presente texto, comparam-se, de uma perspectiva exclusivamente lingüística, duas axiomatizações diferentes, mas logicamente equivalentes, da Gramática Categorial: 1) a chamada Gramática Categorial Clássica e 2) o cálculo de Lambek em Dedução Natural. Para isso, cada uma dessas axiomatizações é apresentada separadamente e depois ambas são consideradas em relação ao tipo de representação do conhecimento lingüístico em cada uma delas. Por fim, apresenta-se como conclusão a preferência pela axiomatização da Gramática Categorial Clássica. E, ainda 
que ela tenha uma motivação exclusivamente lingüística, a diferença entre as duas pode afetar a maneira como o conhecimento lingüístico (especialmente o lexical) precisa ser representado em analisadores para Gramática Categorial.

Palavras-chave: Gramática Categorial; cálculo de Lambek; representação do conhecimento lingüístico.

\begin{abstract}
In the present essay, two different but logically equivalent axiomatizations of Categorial Grammar are compared from an exclusively linguistic perspective: 1 ) the so called Classical Categorial Grammar, and 2) the Lambek calculus in Natural Deduction. In order to do that, each axiomatization are presented separately, and both versions are commented in relation to the type of linguitic knowledge representation for which they allow. In the conclusion, the Classical Categorial Grammar is argued to be the best option. Although it is for an exclusively linguistic reason, it can affect the way that linguistic knowledge (especially lexical knowledge) is represented in any parser for Categorial Grammar.
\end{abstract}

Key-words: Categorial Grammar; Lambek calculus; linguistic knowledge representation.

\title{
REFERÊNCIAS
}

AJDUKIEWICZ, K. Die syntaktische Konnexität. Studia Philosophica, v. 1, p. 1-27, 1935.

BAR-HILLEL, Y. A quasi-arithmetical notation for syntactic description. Language, v. 29, p. 47-58, 1953.

BORGES NETO, J. Introdução às gramáticas categoriais. Curitiba, 1999.

CARPENTER, B. Type-logical semantics. Cambridge, Mass.: The MIT Press, 1997.

COHEN, J. M. The equivalence of two concepts of categorial grammar. Information and Control, v. 10, p. 475-484, 1967.

MOORTGAT, M. Categorial investigations: logical and linguistic aspects of the Lambek calculus. Dordrecht: Foris, 1988.

MORRILL, G. V. Type logical grammar - categorial logic of signs. Dordrecht: Kluwer, 1994.

STEEDMAN, M. Combinators and grammars. In: language structures. Dordrecht: Reidel, 1988. p. 417-442. Categorial grammars and natural

WOOD, M. M. Categorial grammars. London: Routledge, 1993. 